\title{
On the Torsion Function with Mixed Boundary Conditions
}

\section{M. van den Berg ${ }^{1} \cdot$ Tom Carroll $^{2}$}

Received: 5 November 2019 / Accepted: 31 May 2020 / Published online: 16 June 2020

(C) The Author(s) 2020

\begin{abstract}
Let $D$ be a non-empty open subset of $\mathbb{R}^{m}, m \geq 2$, with boundary $\partial D$, with finite Lebesgue measure $|D|$, and which satisfies a parabolic Harnack principle. Let $K$ be a compact, nonpolar subset of $D$. We obtain the leading asymptotic behaviour as $\varepsilon \downarrow 0$ of the $L^{\infty}$ norm of the torsion function with a Neumann boundary condition on $\partial D$, and a Dirichlet boundary condition on $\partial(\varepsilon K)$, in terms of the first eigenvalue of the Laplacian with corresponding boundary conditions. These estimates quantify those of Burdzy, Chen and Marshall who showed that $D \backslash K$ is a non-trap domain.
\end{abstract}

Keywords Torsion function · Dirichlet boundary condition $\cdot$ Neumann boundary condition

Mathematics Subject Classification (2010) $35 \mathrm{~J} 25 \cdot 35 \mathrm{~J} 05 \cdot 35 \mathrm{P} 15$

\section{Introduction and Main Results}

Let $D$ be an open, non-empty set in $\mathbb{R}^{m}, m \geq 2$, with finite Lebesgue measure $|D|$, and let $K \subset D$ be a compact set with boundary $\partial K$, and with positive logarithmic capacity if $m=2$ or with positive Newtonian capacity cap $(K)$ if $m \geq 3$. Let $u_{K, D}$ be the solution of

$$
-\Delta u=1,
$$

with Dirichlet boundary condition

$$
u(x)=0, x \in \partial K,
$$

and Neumann boundary condition

$$
\frac{\partial u}{\partial v}(x)=0, x \in \partial D,
$$

M. van den Berg

mamvdb@bristol.ac.uk

Tom Carroll

t.carroll@ucc.ie

1 School of Mathematics, University of Bristol Fry Building, Woodland Road, Bristol BS8 1UG, UK

2 Department of Mathematics, University College Cork, Cork, Ireland 
where $v$ is the inward normal. Boundary conditions Eqs. 1 and 2 have to be understood in the weak sense. In particular Eq. 1 holds for all regular points of $\partial K$. Let $\pi_{D}(x, y ; t)$, $x \in D, y \in D, t>0$ denote the Neumann heat kernel for $D$. We say that the parabolic Harnack principle (PHP for short) holds in $D$ if for some $t_{0} \in(0, \infty)$ there exists $c_{0}=$ $c_{0}\left(D, t_{0}\right)<\infty$, such that

$$
\pi_{D}(x, y ; t) \leq c_{0} \pi_{D}(v, w ; t), t \geq t_{0}, x, y, v, w \in D .
$$

See also [8]. As was pointed out in [4], PHP is equivalent to the following assertion: there exist $t_{1} \in(0, \infty), c_{1}<\infty, c_{2}>0$ depending on $D$ such that

$$
\sup _{x, y \in D}\left|\pi_{D}(x, y ; t)-\frac{1}{|D|}\right| \leq c_{1} e^{-c_{2} t}, t \geq t_{1} .
$$

It was shown in [4] that if $D$ satisfies PHP then $u_{K, D}$ is bounded, and $D \backslash K$ is a nontrap domain. In Theorem 1 below we quantify this statement in terms of the first eigenvalue $\lambda(K, D)$ of the Laplacian with boundary conditions Eqs. 1 and 2 in the case where $K$ is scaled down by a factor $\varepsilon$ with respect to a fixed point (the origin) in $D$.

Estimates of this type are well known for the torsion function $u_{\Omega}$ for an open set $\Omega$ satisfying a 0 Dirichlet boundary condition on $\partial \Omega$. In [2] it was shown that $u_{\Omega} \in L^{\infty}(\Omega)$ if and only if $\lambda(\Omega)>0$. If the latter holds then

$$
\lambda(\Omega)^{-1} \leq\left\|u_{\Omega}\right\|_{\infty} \leq \mathfrak{c}_{m} \lambda(\Omega)^{-1},
$$

where $\mathfrak{c}_{m}$ is the sharp constant defined by

$$
\mathfrak{c}_{m}=\sup \left\{\lambda(\Omega)\left\|u_{\Omega}\right\|_{\infty}: \Omega \text { open in } \mathbb{R}^{m}, \lambda(\Omega)>0\right\},
$$

and $\|\cdot\|_{p}$ denotes the standard $L^{p}$ norm, $1 \leq p \leq \infty$.

In [2] it was shown that $\mathfrak{c}_{m} \leq 4+3 m \log 2$. This bound has been improved since. See for example [5] and [10]. For general open, non-empty, and connected $D$, and a non-empty compact subset $K \subset D$ one does not have boundedness of $u_{K, D}$. Examples of these trap domains were given in [4].

Theorem 1 Let $D \subset \mathbb{R}^{m}, m \geq 2$, be open, non-empty, containing the origin, and let $D$ satisfy the parabolic Harnack principle. If $K$ is a non-polar compact subset of $D$, then for $\varepsilon \downarrow 0$,

$$
\lambda(\varepsilon K, D)\left\|u_{\varepsilon K, D}\right\|_{\infty}=\left\{\begin{array}{l}
1+O\left(\left(\log \varepsilon^{-1}\right)^{-1 / 2}\right), m=2, \\
1+O\left(\varepsilon^{(m-2) / 2}\right), \quad m \geq 3,
\end{array}\right.
$$

where $\varepsilon K=\left\{y \in \mathbb{R}^{m}: \varepsilon^{-1} y \in K\right\}$. Furthermore for any non-polar compact set $K \subset D$,

$$
\left\|u_{K, D}\right\|_{\infty} \geq \frac{1}{\lambda(K, D)}
$$

It was shown in Theorem 2.5(i) in [4] that if Eq. 3 holds, then the Neumann Laplacian on $D$ has discrete spectrum. Sufficient geometric conditions for $D$ to satisfy the PHP were obtained in, for example, Corollary 2.7 of [4]. Conversely PHP implies some geometric and spectral properties of $D$. The proposition below is of independent interest.

Proposition 2 Let $D$ be open, non-empty, with $|D|<\infty$. If Eq. 3 holds then we have the following.

(i) D is connected.

(ii) The first eigenvalue of the Neumann Laplacian acting in $L^{2}(D)$ has multiplicity 1. 
(iii)

$$
\mu(B)\left(\frac{|B|}{|D|}\right)^{2 / m} \geq \mu(D) \geq c_{2},
$$

where $\mu(D)$ is the first non-zero eigenvalue of the Neumann Laplacian acting in $L^{2}(D)$, and $B$ is a ball of radius 1 in $\mathbb{R}^{m}$.

\section{Proof of Theorem 1}

In this section we prove Theorem 1.

Proof Let $\pi_{K, D}(x, y ; t), x \in D \backslash K, y \in D \backslash K, t>0$ denote the heat kernel with a Neumann boundary condition on $\partial D$, and with a 0 Dirichlet boundary condition on $\partial K$. We have for $\delta \in(0,1)$,

$$
\begin{aligned}
u_{K, D}(x) & =\int_{0}^{\infty} d t \int_{D \backslash K} d y \pi_{K, D}(x, y ; t) \\
& =\int_{0}^{t_{1} /(1-\delta)} d t \int_{D \backslash K} d y \pi_{K, D}(x, y ; t)+\int_{t_{1} /(1-\delta)}^{\infty} d t \int_{D \backslash K} d y \pi_{K, D}(x, y ; t) \\
& \leq \int_{0}^{t_{1} /(1-\delta)} d t \int_{D \backslash K} d y \pi_{D}(x, y ; t)+\int_{t_{1} /(1-\delta)} d t \int_{D \backslash K} d y \pi_{K, D}(x, y ; t) \\
& \leq \frac{t_{1}}{1-\delta}+\int_{t_{1} /(1-\delta)}^{\infty} d t \int_{D \backslash K} d y \pi_{K, D}(x, y ; t) .
\end{aligned}
$$

By the heat semigroup property, and by Cauchy-Schwarz's inequality,

$$
\begin{aligned}
\pi_{K, D}(x, y ; t) & =\int_{D \backslash K} \pi_{K, D}(x, z ; t / 2) \pi_{K, D}(z, y ; t / 2) d z \\
& \leq\left(\int_{D \backslash K} \pi_{K, D}(x, z ; t / 2)^{2} d z\right)^{1 / 2}\left(\int_{D \backslash K} \pi_{K, D}(z, y ; t / 2)^{2} d z\right)^{1 / 2} \\
& =\left(\pi_{K, D}(x, x ; t) \pi_{K, D}(y, y ; t)\right)^{1 / 2} .
\end{aligned}
$$

By the spectral theorem we have

$$
\pi_{K, D}(x, x ; t) \leq e^{-\delta t \lambda(K, D)} \pi_{K, D}(x, x ;(1-\delta) t) .
$$

By Eqs. 8 and 9,

$$
\begin{aligned}
\left(\pi_{K, D}(x, y ; t)\right)^{\delta} & \leq e^{-\delta^{2} t \lambda(K, D)}\left(\pi_{K, D}(x, x ;(1-\delta) t) \pi_{K, D}(y, y ;(1-\delta) t)\right)^{\delta / 2} \\
& \leq e^{-\delta^{2} t \lambda(K, D)} \sup _{x, y \in D}\left(\pi_{K, D}(x, y ;(1-\delta) t)\right)^{\delta} \\
& \leq e^{-\delta^{2} t \lambda(K, D)} \sup _{x, y \in D}\left(\pi_{D}(x, y ;(1-\delta) t)\right)^{\delta} .
\end{aligned}
$$

By Eq. 3,

$$
\begin{aligned}
\left(\pi_{D}(x, y ;(1-\delta) t)\right)^{\delta} & \leq\left(\frac{1}{|D|}+c_{1} e^{-c_{2}(1-\delta) t}\right)^{\delta} \\
& \leq \frac{1}{|D|^{\delta}}+c_{1}^{\delta} e^{-c_{2} \delta(1-\delta) t}, t \geq \frac{t_{1}}{1-\delta}
\end{aligned}
$$


This, together with Eq. 10, gives

$$
\left(\pi_{K, D}(x, y ; t)\right)^{\delta} \leq e^{-\delta^{2} t \lambda(K, D)}\left(\frac{1}{|D|^{\delta}}+c_{1}^{\delta} e^{-c_{2} \delta(1-\delta) t}\right), t \geq \frac{t_{1}}{1-\delta} .
$$

We obtain by Eq. 11, and by Hölder's inequality,

$$
\begin{aligned}
& \int_{t_{1} /(1-\delta)}^{\infty} d t \int_{D \backslash K} d y \pi_{K, D}(x, y ; t) \\
\leq & \int_{t_{1} /(1-\delta)}^{\infty} d t \int_{D \backslash K} d y\left(\pi_{K, D}(x, y ; t)\right)^{1-\delta} e^{-\delta^{2} t \lambda(K, D)}\left(\frac{1}{|D|^{\delta}}+c_{1}^{\delta} e^{-c_{2} \delta(1-\delta) t}\right) \\
\leq & \int_{t_{1} /(1-\delta)}^{\infty} d t \int_{D} d y\left(\pi_{D}(x, y ; t)\right)^{1-\delta} e^{-\delta^{2} t \lambda(K, D)}\left(\frac{1}{|D|^{\delta}}+c_{1}^{\delta} e^{-c_{2} \delta(1-\delta) t}\right) \\
\leq & \int_{t_{1} /(1-\delta)}^{\infty} d t\left(\int_{D} d y \pi_{D}(x, y ; t)\right)^{1-\delta}|D|^{\delta} e^{-\delta^{2} t \lambda(K, D)}\left(\frac{1}{|D|^{\delta}}+c_{1}^{\delta} e^{-c_{2} \delta(1-\delta) t}\right) \\
= & \frac{1}{\delta^{2} \lambda(K, D)} e^{-\delta^{2} t_{1} \lambda(K, D) /(1-\delta)} \\
& +c_{1}^{\delta}|D|^{\delta}\left(c_{2} \delta(1-\delta)+\delta^{2} \lambda(K, D)\right)^{-1} e^{-t_{1}\left(\delta c_{2}+\delta^{2} \lambda(K, D) /(1-\delta)\right)} \\
\leq & \frac{1}{\delta^{2} \lambda(K, D)}+\frac{c_{1}^{\delta}|D|^{\delta}}{c_{2} \delta(1-\delta)} .
\end{aligned}
$$

By Eqs. 7 and 12,

$$
u_{K, D}(x) \lambda(K, D) \leq \delta^{-2}+\left(\frac{t_{1}}{1-\delta}+\frac{c_{1}^{\delta}|D|^{\delta}}{c_{2} \delta(1-\delta)}\right) \lambda(K, D) .
$$

By taking the supremum over all $x \in D \backslash K$ we obtain

$$
\left\|u_{K, D}\right\|_{\infty} \lambda(K, D) \leq \delta^{-2}+\left(\frac{t_{1}}{1-\delta}+\frac{c_{1}^{\delta}|D|^{\delta}}{c_{2} \delta(1-\delta)}\right) \lambda(K, D) .
$$

Hence for $\delta \in(0,1)$ and $\varepsilon \in(0,1)$,

$$
\left\|u_{\varepsilon K, D}\right\|_{\infty} \lambda(\varepsilon K, D) \leq \delta^{-2}+\left(\frac{t_{1}}{1-\delta}+\frac{c_{1}^{\delta}|D|^{\delta}}{c_{2} \delta(1-\delta)}\right) \lambda(\varepsilon K, D) .
$$

In the lemma below we obtain an upper bound for the rate at which $\lambda(\varepsilon K, D) \downarrow 0$ as $\varepsilon \downarrow 0$.

Lemma 3 If $D$ is open, non-empty in $\mathbb{R}^{m}, m \geq 3$, with $|D|<\infty$, and if $K \subset D$ with cap $(K)>0$ then

$$
\limsup _{\varepsilon \downarrow 0} \varepsilon^{2-m} \lambda(\varepsilon K, D) \leq \frac{\operatorname{cap}(K)}{|D|} .
$$

If $D$ is open, non-empty in $\mathbb{R}^{2}$, with $|D|<\infty$, and if $K \subset D$ has strictly positive logarithmic capacity, then

$$
\underset{\varepsilon \downarrow 0}{\limsup }\left(\log \varepsilon^{-1}\right) \lambda(\varepsilon K, D) \leq \frac{2 \pi}{|D|} .
$$

We note that (i) the constants in the right-hand sides of Eqs. 14 and 15 are well-known and sharp (see for example [7]), (ii) both formulae hold for arbitrary open and connected sets $D$ with $|D|<\infty$, and without any regularity assumptions on $\partial D$. We now choose

$$
\delta=1-|D|^{1 / m} \lambda(\varepsilon K, D)^{1 / 2} .
$$


Then $\delta \in(0,1)$ for all $\varepsilon$ sufficiently small. By Eqs. 13 and 16,

$$
\left\|u_{\varepsilon K, D}\right\|_{\infty} \lambda(\varepsilon K, D) \leq 1+O\left(\lambda(\varepsilon K, D)^{1 / 2}\right) .
$$

The proof of Eq. 5 is similar to the one of Theorem 5 in [3], and Theorem 1, (0.5) in [1]. Let $\psi$ denote the normalised first eigenfunction (positive) of the Laplacian with Neumann and Dirichlet boundary conditions on $\partial D$ and $\partial K$ respectively, suppressing both $K$ and $D$ dependence. We have by Cauchy-Schwarz's inequality that $\int_{D \backslash K} \psi \leq|D \backslash K|^{1 / 2}$. Using

$$
\psi \frac{\partial u_{K, D}}{\partial v}=u_{K, D} \frac{\partial \psi}{\partial v}=0 \text { on } \partial D \cup \partial K,
$$

we obtain by Green's formula,

$$
\begin{aligned}
\lambda(K, D)\left\|u_{K, D}\right\|_{\infty} \int_{D \backslash K} \psi & \geq \lambda(K, D) \int_{D \backslash K} u_{K, D} \psi=-\int_{D \backslash K} u_{K, D} \Delta \psi \\
& =-\int_{D \backslash K} \psi \Delta u_{K, D}=\int_{D \backslash K} \psi .
\end{aligned}
$$

This implies the assertion.

Finally Eq. 4 follows by Eqs. 5, 17, and Lemma 3.

\section{Proof of Lemma 3 and Proposition 2}

Proof of Lemma 3 Recall that $0 \in D$, and so

$$
R=\min \{|y|: y \in \partial D\}>0 .
$$

Since $K$ is compact,

$$
R_{K}=\max \{|x|: x \in K\}<\infty .
$$

Let

$$
\varepsilon_{1}=\min \left\{1, \frac{R}{R_{K}}\right\} .
$$

If $\varepsilon \leq \varepsilon_{1}$ then $\varepsilon K \subset B(0 ; R)$. See [9] for estimates related to the proof of Lemma 3. First we consider the case $m \geq 3$. Let $\mu_{K}$ denote the equilibrium measure of $K$ in $\mathbb{R}^{m}$, and let

$$
\phi_{K}(x)=\frac{\Gamma((m-2) / 2)}{4 \pi^{m / 2}} \int_{K} \mu_{K}(d y)|x-y|^{2-m} .
$$

Then $\phi_{K}(x)=1, x \in K, 0<\phi_{K}<1, x \in \mathbb{R}^{m} \backslash K$, and $\phi_{K}$ is smooth on the complement of $K$. We use $1-\phi_{K}$ as a trial function in the Rayleigh-Ritz characterisation of $\lambda(K, D)$. This gives

$$
\begin{aligned}
\lambda(K, D) & =\inf _{u \in H^{1}(D),\left.u\right|_{K}=0} \frac{\int_{D \backslash K}|\nabla u|^{2}}{\int_{D \backslash K} u^{2}} \\
& \leq \frac{\int_{D \backslash K}\left|\nabla \phi_{K}\right|^{2}}{\int_{D \backslash K}\left(1-\phi_{K}\right)^{2}} \\
& \leq \frac{\int_{\mathbb{R}^{m} \backslash K}\left|\nabla \phi_{K}\right|^{2}}{\int_{D \backslash K}\left(1-\phi_{K}\right)^{2}} \\
& =\frac{\operatorname{cap}(K)}{\int_{D \backslash K}\left(1-\phi_{K}\right)^{2}} .
\end{aligned}
$$


It remains to bound the denominator in the right-hand side of Eq. 18 from below. Since we will apply this lower bound with $\varepsilon_{1} K$ rather than $K$ itself, we assume that $K \subset B(0 ; R)$. We let $0<\alpha<1$. It is a standard fact that the capacitary potential is monotone increasing in $K$. In particular,

$$
\phi_{K}(x) \leq \phi_{B(0 ; R)}(x)=\min \left\{1,\left(\frac{R}{|x|}\right)^{m-2}\right\}
$$

Hence

$$
\begin{aligned}
\int_{D \backslash K}\left(1-\phi_{K}\right)^{2} & \geq(1-\alpha)^{2} \int_{\left\{\phi_{K}(x) \leq \alpha\right\} \cap D} 1 \\
& \geq(1-\alpha)^{2}\left(|D|-\left|\left\{\phi_{B(0 ; R)}(x)>\alpha\right\}\right|\right) \\
& \geq(1-\alpha)^{2}\left(|D|-\alpha^{-m /(m-2)} \omega_{m} R^{m}\right),
\end{aligned}
$$

where $\omega_{m}=\left|B_{1}(0)\right|$. We choose $\alpha$ such that

$$
\alpha=\alpha^{-m /(m-2)} \frac{|B(0 ; R)|}{|D|} .
$$

This, together with Eqs. 18, 19 and 20 implies

$$
\lambda(K, D) \leq \frac{\operatorname{cap}(K)}{|D|}\left(1-\left(\frac{|B(0 ; R)|}{|D|}\right)^{(m-2) /(2(m-1))}\right)^{-3} .
$$

In particular for $\varepsilon \in(0,1], \varepsilon \varepsilon_{1} K \subseteq \varepsilon B(0 ; R)$, and this together with Eq. 21 gives

$$
\lambda\left(\varepsilon \varepsilon_{1} K, D\right) \leq \frac{\operatorname{cap}\left(\varepsilon \varepsilon_{1} K\right)}{|D|}\left(1-\left(\frac{\varepsilon|B(0 ; R)|}{|D|}\right)^{(m-2) /(2(m-1))}\right)^{-3} .
$$

Formula Eq. 14 follows by Eq. 22, and scaling of the Newtonian capacity,

$$
\operatorname{cap}(\varepsilon K)=\varepsilon^{m-2} \operatorname{cap}(K) \text {. }
$$

Next we consider the planar case $m=2$. We use Hadamard's method of descent so as to avoid logarithmic potential theory. See for example p.51 in [9]. Let $h \geq R$, and consider the cylinder $(D \backslash K) \times(0, h) \subset \mathbb{R}^{3}$. Then the first eigenvalue of the Laplacian acting in $\left.L^{2}(D \backslash K)\right)$ with Dirichlet boundary condition on $\partial K$, and Neumann boundary condition on $\partial D$ is precisely equal to the first eigenvalue of the Laplacian acting in $L^{2}((D \backslash K) \times(0, h))$ with Dirichlet boundary condition on $\partial(K \times(0, h))$, and Neumann boundary condition on $\partial(D \times(0, h)) \backslash \partial(K \times(0, h))$. We apply Eq. 21 to the setting above and obtain by monotonicity of Newtonian capacity,

$$
\begin{aligned}
\lambda\left(\varepsilon \varepsilon_{1} K, D\right) & \leq \lambda(\varepsilon B(0 ; R), D) \\
& \leq \frac{\operatorname{cap}(B(0 ; \varepsilon R) \times(0, h))}{|D| h}\left(1-\left(\frac{\varepsilon|B(0 ; R)|}{|D|}\right)^{1 / 4}\right)^{-3} .
\end{aligned}
$$

To obtain an upper bound on cap $(B(0 ; \varepsilon R) \times(0, h))$ we let $C\left(R^{\prime}, h^{\prime}\right) \subset \mathbb{R}^{3}$ be an ellipsoid with a circular cross section of radius $R^{\prime}$ and axis $h^{\prime}$. Then for a suitable translation and rotation $C\left(R^{\prime}, h^{\prime}\right) \supset B(0 ; \varepsilon R) \times(0, h)$ provided

$$
\frac{h^{2}}{h^{\prime 2}}+\frac{(\varepsilon R)^{2}}{R^{\prime 2}} \leq 1
$$

We let $\alpha \in(0,1)$ be arbitrary, and choose

$$
R^{\prime}=\varepsilon^{-\alpha}(\varepsilon R)
$$


and

$$
h^{\prime}=\left(1-\varepsilon^{2 \alpha}\right)^{-1 / 2} h .
$$

The choice Eqs. 25-26 satisfies Eq. 24. For $\frac{h^{\prime}}{R^{\prime}} \rightarrow \infty$, or equivalently $\varepsilon \downarrow 0$ with $h$ fixed, we have by formula (12) on p.260 in [6],

$$
\begin{aligned}
\operatorname{cap}\left(C\left(R^{\prime}, h^{\prime}\right)\right) & =\frac{2 \pi h^{\prime}}{\log \left(h^{\prime} / R^{\prime}\right)}(1+o(1)) \\
& \leq \frac{2 \pi h}{\left(1-\varepsilon^{2 \alpha}\right)^{1 / 2} \log \left(h / R^{\prime}\right)}(1+o(1)) \\
& \leq \frac{2 \pi h}{(1-\alpha)\left(1-\varepsilon^{2 \alpha}\right)^{1 / 2} \log \varepsilon^{-1}}(1+o(1)) .
\end{aligned}
$$

Thus,

By Eq. 23,

$$
\frac{\operatorname{cap}(B(0 ; \varepsilon R) \times(0, h))}{|D| h} \leq \frac{2 \pi}{(1-\alpha)|D| \log \varepsilon^{-1}}(1+o(1)) .
$$

$$
\limsup _{\varepsilon \downarrow 0}\left(\log \varepsilon^{-1}\right) \lambda\left(\varepsilon \varepsilon_{1} K, D\right) \leq \frac{2 \pi}{(1-\alpha)|D|} .
$$

Since $\alpha \in(0,1)$ was arbitrary, this completes the proof of the case $m=2$.

Proof of Proposition 2 To prove (i) we recall that, since $D$ is open, $D$ is a countable union of open components. Suppose that this union contains at least two elements, one of which is $C$. Then both $C$ and $D \backslash C$ are open and non-empty. Let $1_{A}$ denote the indicator function of a set $A$. From Eq. 3 we obtain,

$$
\left|\int_{C} d y \pi_{D}(x, y ; t)-\frac{|C|}{|D|}\right| \leq c_{1}|C| e^{-c_{2} t}, t \geq t_{1}, x \in D .
$$

We note that

$$
q_{C, D}(x ; t)=\int_{C} d y \pi_{D}(x, y ; t)
$$

is the solution of the heat equation

with initial condition

$$
\Delta q=\frac{\partial q}{\partial t}
$$

$$
q(x ; 0)=1_{C}(x),
$$

and with a Neumann (insulating) boundary condition on $\partial D$. It follows that

$$
q_{C, D}(x ; t)=1_{C}(x), t>0 .
$$

From Eq. 3 we have

$$
\left|1-\frac{|C|}{|D|}\right| \leq c_{1}|C| e^{-c_{2} t}, t \geq t_{1}, x \in C .
$$

We conclude that, by taking the limit $t \rightarrow \infty,|C|=|D|$. Since $C \subset D,|D \backslash C|=0$. This contradicts $D \backslash C$ is open and non-empty. This in turn implies that $D$ consists of just one component $C$. Hence $C$ is connected. This implies assertion (ii). To prove (iii) we have that Eq. 3 implies

$$
\int_{D} d x \pi_{D}(x, x ; t) \leq 1+c_{1}|D| e^{-c_{2} t}, t \geq t_{1}
$$


Hence the Neumann heat semigroup is trace-class, and

$$
1+e^{-t \mu(D)} \leq \int_{D} d x \pi_{D}(x, x ; t) \leq 1+c_{1}|D| e^{-c_{2} t}, t \geq t_{1} .
$$

Taking the limit $t \rightarrow \infty$ in Eq. 27 implies the second inequality in Eq. 6. The first inequality in Eq. 6 is due to Weinberger [11].

Acknowledgments MvdB acknowledges support by the Leverhulme Trust through Emeritus Fellowship EM-2018-011-9.

Open Access This article is licensed under a Creative Commons Attribution 4.0 International License, which permits use, sharing, adaptation, distribution and reproduction in any medium or format, as long as you give appropriate credit to the original author(s) and the source, provide a link to the Creative Commons licence, and indicate if changes were made. The images or other third party material in this article are included in the article's Creative Commons licence, unless indicated otherwise in a credit line to the material. If material is not included in the article's Creative Commons licence and your intended use is not permitted by statutory regulation or exceeds the permitted use, you will need to obtain permission directly from the copyright holder. To view a copy of this licence, visit http://creativecommons.org/licenses/by/4.0/.

\section{References}

1. Bañuelos, R., Carroll, T.: Brownian motion and the fundamental frequency of a drum. Duke Math. J. 75, 575-602 (1994)

2. van den Berg, M., Carroll, T.: Hardy inequality and $L^{p}$ estimates for the torsion function. Bull. Lond. Math Soc. 41, 980-986 (2009)

3. van den Berg, M.: Estimates for the torsion function and Sobolev constants. Potential Anal. 36, 607-616 (2012)

4. Burdzy, K., Chen, Z.-Q., Marshall, D.E.: Traps for reflected Brownian motion. Math. Z. 252, 103-132 (2006)

5. Giorgi, T., Smits, R.G.: Principal eigenvalue estimates via the supremum of torsion. Indiana Univ. Math. J. 59, 987-1011 (2010)

6. Itô, K., McKean, H.P.: Diffusion processes and their sample paths. Second printing, corrected. Die Grundlehren der mathematischen Wissenschaften, Band 125. Springer-Verlag, Berlin-New York (1974)

7. Ozawa, S.: The first eigenvalue of the Laplacian on two-dimensional Riemannian manifolds. Tohoku Math. J. 34, 7-14 (1982)

8. Saloff-Coste, L.: Precise estimates on the rate at which certain diffusions tend to equilibrium. Math. Z. 217, 641-677 (1994)

9. Taylor, M.E.: Estimate on the fundamental frequency of a drum. Duke Math. J. 46, 447-453 (1979)

10. Vogt, H.: $L_{\infty}$ - estimates for the torsion function and $L_{\infty}$-growth of semigroups satisfying Gaussian bounds. Potential Anal. 51, 37-47 (2019)

11. Weinberger, H.F.: An isoperimetric inequality for the $N$-dimensional free membrane problem. J. Rational Mech. Anal. 5, 633-636 (1956)

Publisher's Note Springer Nature remains neutral with regard to jurisdictional claims in published maps and institutional affiliations. 